\title{
Pseudomonas eradication in cystic fibrosis: who will join the ELITE?
}

\section{Alan R Smyth}

Arguably, the most significant event in the life of a person with cystic fibrosis (CF) is the acquisition of chronic pulmonary infection with Pseudomonas aeruginosa. It heralds, in many cases, more hospitalisations, ${ }^{1}$ a more rapid decline in pulmonary function and a shorter life expectancy. ${ }^{2} P$ aeruginosa has the ability to form biofilms which are composed of nonmotile organisms embedded in an exopolysaccharide matrix and which confer resistance to antibiotics. ${ }^{3}$ In individuals with CF, biofilms have been observed in lungs examined at autopsy and explanted lungs from transplant recipients. ${ }^{4}$ The extraordinary persistence of infection with $P$ aeruginosa in the CF lung is thought to derive from biofilm formation. The mucoid phenotype of $P$ aeruginosa is a marker of biofilm formation, ${ }^{4}$ and this phenotype is in turn associated with a worse prognosis compared with nonmucoid organisms. ${ }^{5}$ However, in the initial stages of infection when the organism is mainly in planktonic form, there is an opportunity to eradicate the infection before it becomes persistent.

The first randomised controlled trial of an eradication regimen for early $P$ aeruginosa infection was reported by Valerius and Koch in 1991. ${ }^{6}$ This evaluated a 3week regimen of nebulised colistin and oral ciprofloxacin (compared with no treatment) and found that fewer patients developed chronic infection in the active group over a period of 2 years. A further study using historical controls suggested that a longer course of colistin and ciprofloxacin ( 3 months) achieved a longer period free of recurrent infection with $P$ aeruginosa. ${ }^{7}$ Nebulised tobramycin has been compared with placebo in two randomised controlled trials which have shown successful short-term eradication of $P$ aeruginosa from bronchoalveolar

Correspondence to Dr Alan R Smyth, Associate Professor \& Reader in Child Health, Division of Child Health, \& Nottingham Respiratory Biomedical Research Unit, E Floor, East Block, Queens Medical Centre,

Nottingham NG7 2UH UK; alan.smyth@nottingham.ac.uk lavage specimens ${ }^{8}$ and from throat or sputum cultures. ${ }^{9}$ A comparison of nebulised colistin plus ciprofloxacin with nebulised tobramycin was underpowered and showed no difference between groups. ${ }^{10}$ Indeed, there have been few studies which have compared alternative regimens or durations of treatment. The ELITE study ${ }^{11}$ by Ratjen et al reported in this issue of Thorax is therefore a welcome addition to the evidence base for eradication treatment (see page 286).

ELITE incorporates an innovative study design whereby patients who were free of $P$ aeruginosa for at least a year received eradication treatment with nebulised tobramycin for 28 days. At the time of enrolment, serum samples were taken for analysis for antibodies to Pseudomonas exoenzymes. These results were available by the end of the initial 28 days of treatment, and those patients who were antibody positive (suggesting chronic infection) were excluded from randomisation. Antibody-negative patients were randomised to either stop eradication therapy or to have a further 28 days of treatment. The primary outcome (time to recurrence of infection with $P$ aeruginosa) was approximately 26 months in both groups. Secondary outcomes-including the proportion of patients in whom $P$ aeruginosa was eradicated initially, spirometry and nutritional indicesshowed no significant differences between groups. Reported adverse effects and measures of renal function and hearing did not differ between groups. The primary outcome was evaluable in 65 patients, less than the estimated sample size of 100 patients randomised. A shorter course of eradication therapy offers clear advantages for the CF team, the patients and their families. However, the investigators' conclusion that there is no difference between the 28-day and 56-day regimens would be more robust if they had employed an equivalence study design. ${ }^{12}$

So, where next for Pseudomonas eradication? Eradication therapy with either a colistin/ciprofloxacin regimen or with nebulised tobramycin is recommended standard care in European CF centres. ${ }^{13} \mathrm{~A}$ Cochrane review concluded that eradication is achieved, at least initially, but does not recommend one regimen over another. ${ }^{14}$ The ELITE study has provided the first randomised comparison of eradication regimens of different durations. Studies of eradication regimens comparing different drugs or different approaches to drug administration are badly needed. A number of important studies are ongoing or are due to report their findings in the near future.

The recently completed EPIC study has randomised over 300 children with newly acquired Pseudomonas infection to either culture-directed therapy or quarterly cycles of inhaled tobramycin (28 days of tobramycin followed by 56 days off treatment) for 18 months. Patients in each group were further randomised to receive 14 days of either ciprofloxacin or placebo orally-again either directed by culture or in quarterly cycles. ${ }^{15}$ The results of the trial will be published within the next year. The Australasian Cystic Fibrosis Bronchoalveolar Lavage (ACFBAL) study ${ }^{16}$ takes the concept of culture-directed therapy a stage further by randomising children to receive either a single bronchoalveolar lavage (BAL) at 5 years of age, or BAL at baseline, a further BAL specimen to confirm any upper respiratory isolates of $P$ aeruginosa, BAL with intravenous antibiotics and a final specimen at 5 years of age. In both groups a robust eradication regimen ( 2 weeks intravenous antibiotics, 4 weeks ciprofloxacin and 8 weeks nebulised tobramycin) is given where isolates are positive for $P$ aeruginosa. Again, the results are expected within a year. In contrast, the TORPEDO study (http:// WWw.controlled-trials.com/ISRCTN0 2734162/TORPEDO-CF), which will commence shortly in the UK, will compare 2 weeks of intravenous antibiotics with 3 months of oral ciprofloxacin, both combined with 3 months of nebulised colistin.

It has been a major achievement of the last two decades of CF care to make eradication treatment for $P$ aeruginosa routine. Eradication, along with meticulous microbiological surveillance and measures to prevent cross-infection, has reduced the median prevalence of chronic Pseudomonas infection to around 11\% in children with CF attending UK centres. ${ }^{17}$ The ELITE study is the first step in determining the optimal eradication regimen. Optimal therapy will be of the shortest duration compatible with 
long-term eradication, will minimise drug side effects and antibiotic resistance, but with the least possible upset to schooling, work and family life. Getting there will require every CF centre to participate in multicentre trials and the timely application of evidence to clinical practice. Who will join the ELITE?

Competing interests ARS is Director of the Trent Local Children's Research Network, part of the UK NIHR Medicines for Children Network.

Provenance and peer review Commissioned; not externally peer reviewed.

Thorax 2010;65:281-282

doi:10.1136/thx.2009.130708

\section{REFERENCES}

1. Nixon GM, Armstrong DS, Carzino R, et al. Clinical outcome after early Pseudomonas aeruginosa infection in cystic fibrosis. J Pediatr 2001;138:699-704.

2. Emerson J, Rosenfeld M, McNamara S, et al. Pseudomonas aeruginosa and other predictors of mortality and morbidity in young children with cystic fibrosis. Pediatr Pulmonol 2002;34:91-100.
3. Drenkard E. Antimicrobial resistance of Pseudomonas aeruginosa biofilms. Microbes Infect 2003;5:1213-19.

4. Bjarnsholt T, Jensen PO, Fiandaca MJ, et al. Pseudomonas aeruginosa biofilms in the respiratory tract of cystic fibrosis patients. Pediatr Pulmonol 2009:44:547-58.

5. Li Z, Kosorok MR, Farrell PM, et al. Longitudinal development of mucoid Pseudomonas aeruginosa infection and lung disease progression in children with cystic fibrosis. JAMA 2005;293:581-8.

6. Valerius N, Koch CHN. Prevention of chronic Pseudomonas aeruginosa colonisation in cystic fibrosis by early treatment. Lancet 1991;338:725-6.

7. Hoiby N, Frederiksen B, Pressler T. Eradication of early Pseudomonas aeruginosa infection. J Cyst Fibros 2005;4 Suppl 2:49-54.

8. Gibson RL, Emerson J, McNamara S, et al. Significant microbiological effect of inhaled tobramycin in young children with cystic fibrosis. Am J Respir Crit Care Med 2003:167:841-9.

9. Wiesemann HG, Steinkamp G, Ratjen F, et al. Placebo-controlled, double-blind, randomized study of aerosolized tobramycin for early treatment of Pseudomonas aeruginosa colonization in cystic fibrosis. Pediatr Pulmonol 1998;25:88-92.

10. Proesmans M, Boulanger L, Vermeulen F, et al. Eradication of recent Pseudomonas aeruginosa isolation: TOBI versus colistin/ciprofloxacin. J Cyst Fibros 2008:7:S64.
11. Ratjen F, Munck A, Kho P, et al, for the ELITE study group. Treatment of early Pseudomonas aeruginosa infection in patients with cystic fibrosis: the ELITE trial. Thorax 2010;65:286-91.

12. Jones $\mathbf{B}$, Jarvis $P$, Lewis JA, et al. Trials to assess equivalence: the importance of rigorous methods. BMJ 1996;313:36-9.

13. Doring G, Conway SP, Heijerman HGM, et al. Antibiotic therapy against Pseudomonas aeruginosa in cystic fibrosis: a European consensus. Eur Respir J 2000;16:749-67.

14. Langton Hewer SC, Smyth AR. Antibiotic strategies for eradicating Pseudomonas aeruginosa in people with cystic fibrosis. Cochrane Database Syst Rev 2009; (4):CD004197.

15. Treggiari MM, Rosenfeld M, Mayer-Hamblett N, et al. Early anti-pseudomonal acquisition in young patients with cystic fibrosis: rationale and design of the EPIC clinical trial and observational study. Contemp Clin Trials 2009;30:256-68

16. Cheney J, Vidmar S, Grimwood K, et al. Interim outcomes of a Pseudomonas aeruginosa $(\mathrm{Pa})$ eradication protocol in young children in the Australasian Cystic Fibrosis Bronchoalveolar Lavage (ACFBAL) study. J Cyst Fibros 2009;8:S39.

17. UK Cysic Fibrosis Trust. UK CF Registry. Annual Data Report 2008. Bromley: Cystic Fibrosis Trust, 2009

\section{Is postmenopausal HRT a risk factor for adult-onset asthma?}

\section{A E Tattersfield}

Adult onset asthma is often progressive ${ }^{1}$ and the cause of considerable morbidity. Some asthma developing de novo in adults can be attributed to environmental factors, such as occupational sensitisers. When such an association is recognised, asthma can often be prevented, so searching for aetiological factors is well worthwhile. Hormone replacement therapy (HRT) may be one such factor.

Postmenopausal HRT was used widely until recently, being seen as the way for women not only to appear more youthful and glamorous following the menopause but also to be healthier. Various observational studies had suggested a reduced risk of diseases such as osteoporosis, heart disease and possibly dementia. However, when the results of prospective studies became available the picture was, alas, rather less positive. Not only did these studies fail to confirm many of the alleged

Correspondence to Professor A E Tattersfield University of Nottingham, Clinical Sciences Building, Nottingham University Hospital (City campus), Hucknall Road, Nottingham NG5 1PB, UK: anne.tattersfield@nottingham.ac.uk benefits of HRT, they suggested or confirmed important adverse effects including an increased risk of breast cancer, ovarian cancer, venous thrombo-embolism, heart disease, cognitive decline and, more recently, mortality from lung cancer. ${ }^{2-8}$ Should late-onset asthma be on this list?

Cross-sectional studies have shown an association between the use of HRT and a diagnosis of asthma and asthma symptoms. ${ }^{9-11}$ The studies have varied in design and in how asthma was diagnosed, but the findings have been fairly consistent in showing a modest association between HRT use and reported asthma, with ORs ranging from 1.38 to $1.57 .^{9-11}$ The associations with symptoms and asthma medication were of similar magnitude. ${ }^{9-11}$ Two studies found the strongest association in never smokers 911 and two showed an interaction with body mass index (BMI), in that the effects of HRT were seen largely in women with a low BMI. ${ }^{9} 10$ Having a high BMI per se was associated with asthma, ${ }^{9}$ but there was no additional effect from HRT in these more overweight women. Crosssectional studies have limitations, and none of these studies looked at the type of HRTused, nor did they distinguish between new-onset and established asthma.

Data from the large US Nurses Health Study were published in $1995^{12}$ and $2004 .^{13}$ This was the first prospective study to look at the relationship of postmenopausal hormone use to the development of new cases of physician-diagnosed asthma, using biennial questionnaires to determine HRT use. The analyses covered a quarter ${ }^{12}$ and half ${ }^{13}$ a million personyears of follow-up, respectively, with some overlap for the middle period. Both analyses showed an increased risk of developing asthma in association with current use of HRT, and this was similar for oestrogen alone and oestrogen/ progestin preparations. The increased risk with unopposed oestrogen showed a dose-response relationship in the first study. ${ }^{12}$ In the more recent study, by Barr et $a l^{13}$ the rate ratio for newly diagnosed asthma amongst current users of oestrogen only was 2.29 (95\% CI 1.59 to 3.29 ), and this tended to be greater in women with a low BMI. HRT was not a risk factor for developing chronic obstructive pulmonary disease. ${ }^{13}$

This issue of Thorax contains a further prospective study, from Romieu et al (see page 292), looking at the relationship of HRT use to new-onset asthma amongst postmenopausal women in the E3N study, a cohort of 98995 French women using 